\title{
THE OVERLAP APPROACH TOWARD THE DERIVATION OF PHOTOGRAPHIC STELLAR COORDINATES
}

\author{
W.D. GOOGE, C.F. LUKAC, and H. EICHHORN
}

\section{Introduction}

The use of the conditions imposed by the appearance of one star on more than one photographic plate to strengthen the determination of the relationship between the measurement coordinate systems and the system of celestial right ascension and declination, was first advocated by Eichhorn (1960), and since then various reduction procedures have been proposed to incorporate these conditions in the plate-reduction process (Eichhorn, 1963; Henriksen, 1964; Lacroute, 1964; Googe, 1967; Clube, 1967). During the last several years at the Army Map Service the overlap method has been developed and used in several astrometric projects, and the purpose of this paper is to summarize the main features of the algorithm and some of the projects to which it has been applied.

\section{The Algorithm}

Two different types of observations enter into the reductions, the celestial coordinates of the reference stars and the rectangular coordinates of all the stars measured on the plate, and for each type observation equations are formed. For the plate measurements $(x, y)$ the equations are:

$$
\begin{aligned}
& x=a+b \xi+c \eta+\cdots \\
& y=a^{\prime}+b^{\prime} \xi+c^{\prime} \eta+\cdots,
\end{aligned}
$$

where $a, b, c, a^{\prime}, b^{\prime}, c^{\prime}, \ldots$ are the usual plate constants and

$$
\begin{aligned}
& \xi=\xi(\alpha, \delta) \\
& \eta=\eta(\alpha, \delta)
\end{aligned}
$$

are the star's standard coordinates and thus functions of its celestial coordinates $(\alpha, \delta)$. The number and form of the higher-order terms in the expansion depend, of course, on the attributes of the particular reduction being attempted, reflecting as they must the various physical phenomena that enter into the image formation and mensuration processes. The unknowns in these equations are the plate constants and the stellar coordinates. 
A system of equations of the measurement type alone would be singular, that is, it would possess an infinity of least-squares solutions. To remove this singularity as well as to introduce the information contained in the observations of the referencestar coordinates, additional equations are used:

$$
\begin{aligned}
& \alpha=\alpha \\
& \delta=\delta .
\end{aligned}
$$

These are interpreted to mean that the quantities on the left are observations of the unknown true coordinates on the right. In principle, there is no reason why these equations could not be made more elaborate. E.g., if several catalogues of reference material were to be used and there were doubts about the uniformity of systems, additional unknowns could be introduced to parameterize the systematic differences.

It should be noted explicitly that there will be a pair of equations for every measured image of a star and another pair for every reference-star observation. Thus the algorithm applies regardless of the pattern of the photography - a star may appear on one plate or many.

Once the observation equations are formulated, the standard non-linear leastsquares methods apply. The equations are linearized, weighted, formed into a system of normal equations and a solution calculated. In practice, it is wise to eliminate algebraically the star unknowns in order to produce a system of reduced normal equations containing only plate constant unknowns (Googe, 1967).

If many plates are to be included, the system of equations will be large and some care must be exercised to obtain an acceptable solution. Several iterative methods have been discussed (Eichhorn, 1960; Lacroute, 1964; Henriksen, 1964, 1967) and there are many others available (Varga, 1962; Faddeev and Faddeeva, 1963). In particular, the results of Lacroute (1967) using block Jacobi iteration are most encouraging. It should be remembered, however, that the efficiency of these methods depends on the 'condition' of the coefficient matrix which is a delicate function of the number and type of terms used in the plate constant expansion, on the number and distribution of the reference stars, on the overlap pattern, and on the weighting scheme used. Thus one must be cautious about drawing general conclusions concerning speeds of convergence. At the Army Map Service we have preferred exact methods, that is, some form of direct Gaussian elimination. These not only avoid the problem of convergence but supply useful information about the propagation of errors from one plate to another, e.g. the inverse of the normal equations.

Another problem is the error introduced by the linearization of the non-linear observation equations. Our experience has shown that this is non-existent if care is taken to choose sufficiently accurate initial estimates of the unknowns. In fact, we have iterated many overlap adjustments and in every case the changes computed after the second linearization were negligible. 


\section{Zonal Reductions}

At the Army Map Service we are currently at work preparing several zones of photographic material for reduction with the overlap algorithm. One of these zones, consisting of a series of 90 plates exposed for us at the Sydney Observatory by Dr. H. Wood, covers the Southern sky from $-48^{\circ}$ to $-54^{\circ}$. These plates have been measured at the University of South Florida. The region from $-60^{\circ}$ to $-70^{\circ}$ covered by Yale plates will also be reduced in the rigorous manner as will be the South polar cap $\left(-70^{\circ}\right.$ to $\left.-90^{\circ}\right)$.

As preparation for this work we have developed programs to reduce all or part of the plates for a zone taken in the usual edge to centre overlap pattern. In order to test the process we have used measurements supplied by Dr. Stoy for the Cape Photographic Zone from $-52^{\circ}$ to $-56^{\circ}$. By making use of the greater flexibility allowed in the expansion by the overlap redundancy, it was possible to reduce the residual root mean square considerably. Table 1 gives some results from typical overlap solutions performed to evaluate various models.

\section{Table 1}

\begin{tabular}{clc} 
Solution & \multicolumn{1}{c}{ Terms in $x$} & $\begin{array}{c}\text { Residual Root } \\
\text { Mean Square }\end{array}$ \\
1 & $1, \xi$ & $" .253$ \\
2 & $1, \xi, \eta, \xi^{2}, \xi \eta$ & .234 \\
3 & $1, \xi, \eta, \xi^{2}, \xi \eta, \eta^{2}$ & $" 229$ \\
4 & $1, \xi, \eta, \xi^{2}, \xi \eta, \xi\left(\xi^{2}+\eta^{2}\right)$ & $" 195$ \\
5 & $1, \xi, \eta, \xi^{2}, \xi \eta, \eta^{2},\left(\xi^{2}+\eta^{2}\right)$ & $" .194$
\end{tabular}

The first three solutions showed considerable systematic error as well as a high r.m.s. value. In particular, the first adjustment produced plate constants which in the extreme gave systematic errors of $4 / 10$ of a second of arc.

It is perhaps of interest that for a complete zone with about 40000 equations in 20000 unknowns the overlap adjustment takes less than 2 hours on the IBM 7094 computer. This includes the combination of direct and reverse raw measurements, application of comparator calibration corrections, classical solution to determine initial values for the plate constants together with the formation and solution of the linearized observation and normal equations.

\section{Pleiades catalogue}

Another application made of the overlap technique at the Army Map Service has been the reduction of a series of exposures made of the region of the Pleiades cluster 
(Lukac, 1967). Thirteen plates were taken with the 26-inch McCormick telescope at the University of Virginia, and one additional plate was exposed on the 20-inch refractor of the Van Vleck Observatory. All together there were 65 exposures. A diffraction grating was used and the 20000 stellar images produced were measured by Eichhorn. Since only about 500 stars are involved, these abundantly redundant data are being used not only for a thorough statistical evaluation of the overlap algorithm but for the investigation of the diffraction-image formation process. The more complete model allowed by the extra redundancy introduced through the overlap conditions has reduced the systematic errors by a factor of about $\frac{1}{2}$. The accuracy of the resulting stellar coordinates will be of the order of $1 / 100$ of a second of arc.

\section{Advantages and Disadvantages of the Overlap}

The main reason for using the overlap conditions in the plate reduction equations is to strengthen the determination of the relationship between the rectangular and spherical coordinate systems and practical results have verified that this is indeed accomplished. This means that fewer reference stars are needed, and the results of Eichhorn and Gatewood (1967) working with the Northern Hyderabad Zone of the Astrographic Catalogue show that, for this material at least, a decrease of $\frac{2}{3}$ in the number of reference-star observations gives an accuracy comparable to the classical method. At the Army Map Service we have found it possible to reduce accurately some plates in a complex with no reference stars whatever.

The overlap method, naturally, cannot increase the accuracy of the measurements. Nevertheless, putting more information into the reduction allows a more complete model of the image-formation process. This results in a less disparate adjustment. This is one reason why a correct model is more necessary for an overlap adjustment than for a classical solution. But a much more important reason remains. The overlap algorithm has the effect of fusing the plates into a single unit into which unaccounted for distortions can not only propagate but expand and be magnified. Examples of this are given by Eichhorn et al. (1967) and many more could be related. This need for a completely adequate model can only be met by extremely careful statistical and physical analyses.

\section{References}

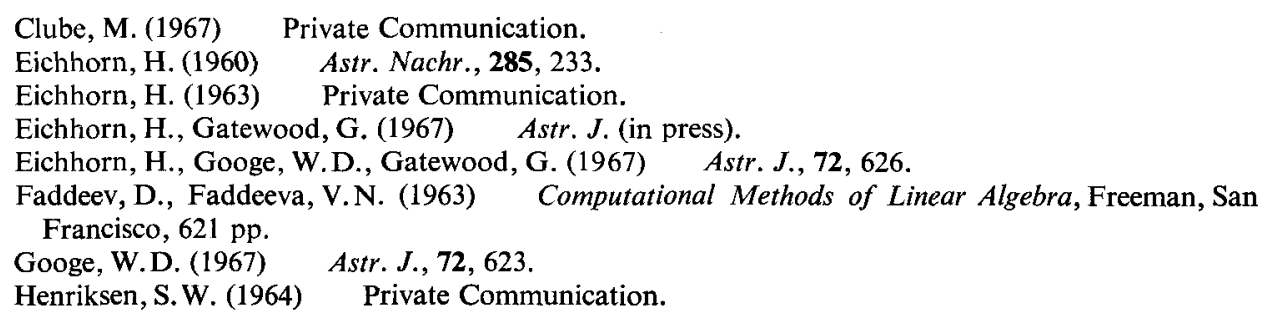


Henriksen, S.W. (1967) Astr. J., 72, 603.

Lacroute, P. (1964) Ann. Obs. Strasbourg, 6.

Lacroute, P. (1967) Private Communication.

Lukac, C.F. (1967) Astr. J., 72, 620.

Varga, R. (1962) Matrix Iterative Analysis, Prentice-Hall, Englewood Cliffs, N.J. 